INPLASY

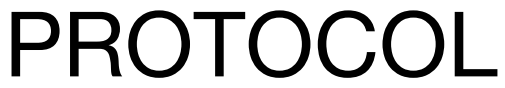

To cite: Chen et al. Effects of traditional Chinese exercise on the treatment of COVID-19: a protocol for a systematic review. Inplasy protocol 202210089. doi:

10.37766/inplasy2022.1.0089

Received: 17 January 2022

Published: 17 January 2022

Corresponding author:

Li Chen

lizi5225@qq.com

Author Affiliation:

Chengdu Sport University.

Support: 18YJC890011.

Review Stage at time of this submission: Preliminary

searches.

Conflicts of interest:

None declared.

\section{Effects of traditional Chinese exercise on the treatment of COVID-19: a protocol for a systematic review}

\author{
Chen, L1; Ji, Y2; Wang, QP3; Chen, P4.
}

Review question / Objective: Since the end of December 2019, COVID-19 has caused a huge impact in many countries and has attracted great attention from countries around the world. In fact, many studies have shown that during the fight against the COVID-19 epidemic. Chinese traditional exercise play an active role in promoting human health. The main purpose of this study is to provide a reliable method and credible evidence to improve the prognosis of patients with COVID-19 through traditional Chinese exercise.

Condition being studied: In recent years, a large number of studies have shown that traditional Chinese sports, as the traditional culture of the Chinese nation, have played a huge role in this new crown epidemic. Therefore, we will study the effect of traditional Chinese exercise on the prognosis and treatment effect of COVID-19 patients in a systematic review and analysis.

INPLASY registration number: This protocol was registered with the International Platform of Registered Systematic Review and Meta-Analysis Protocols (INPLASY) on 17 January 2022 and was last updated on 17 January 2022 (registration number INPLASY202210089).

\section{INTRODUCTION}

Review question / Objective: Since the end of December 2019, COVID-19 has caused a huge impact in many countries and has attracted great attention from countries around the world. In fact, many studies have shown that during the fight against the COVID-19 epidemic. Chinese traditional exercise play an active role in promoting human health. The main purpose of this study is to provide a reliable method and credible evidence to improve the prognosis of patients with COVID-19 through traditional Chinese exercise.

Condition being studied: In recent years, a large number of studies have shown that 
traditional Chinese sports, as the traditional culture of the Chinese nation, have played a huge role in this new crown epidemic. Therefore, we will study the effect of traditional Chinese exercise on the prognosis and treatment effect of COVID-19 patients in a systematic review and analysis.

\section{METHODS}

Search strategy: The following electronic databases will be searched to identify relevant research: PubMed, EMBASE, MEDLINE, Cochrane Library, Web of Science, China National Knowledge Infrastructure Database (CNKI), China Biomedical Literature Database (CBM), China Science and Wan-Fang Database. Search dates: from inception dates to December 2021.

Participant or population: Regardless of gender, age, race, or education and economic status, COVID-19 patients who have been clearly diagnosed and are in recovery will be included. Postoperative infections, psychiatric patients, severe pneumonia or patients unable to exercise due to other reasons will not be included.

Intervention: The experimental group will be divided into 4 groups, treated with TCE, including Wuqinxi, Baduanjin, Yijinjing, Tai Chi, and so on. The control group is treated with non-TCE or pharmacotherapies. Pharmacotherapies include drugs recommended in international or domestic authorized clinical guidelines. Studies which combine TCE with pharmacotherapy are required to use the same pharmacotherapy in both the experimental and the control groups.

Comparator: Comparisons include rest, psychosocial therapy, and drug therapy. In addition, the study will also include studies that compare traditional Chinese exercise therapy with another method of treatment alone.

Study designs to be included: We will include research related to traditional Chinese exercise therapy for COVID-19 patients. In order to obtain a more objective and true evaluation of the research, all the referenced documents must meet the following four conditions at the same time: (1) Published documents with complete documents data; (2) The research subject is confirmed to have COVID-19; (3) The intervention group received traditional Chinese exercise therapy intervention for at least a period of treatment; (4) Including randomized controlled trials (RCT), controlled (nonrandomized) clinical trials (CCT) or cluster.

Eligibility criteria: Participants: Regardless of gender, age, race, or education and economic status, COVID-19 patients who have been clearly diagnosed and are in recovery will be included. Postoperative infections, psychiatric patients, severe pneumonia or patients unable to exercise due to other reasons will not be included.Interventions: The experimental group will be divided into 4 groups, treated with TCE, including Wuqinxi, Baduanjin, Yijinjing, Tai Chi, and so on. The control group is treated with non-TCE or pharmacotherapies. Pharmacotherapies include drugs recommended in international or domestic authorized clinical guidelines. Studies which combine TCE with pharmacotherapy are required to use the same pharmacotherapy in both the experimental and the control groups.Comparators: Comparisons include rest, psychosocial therapy, and drug therapy. In addition, the study will also include studies that compare traditional Chinese exercise therapy with another method of treatment alone.Outcomes: Primary outcomes: The disappearance of the main symptoms (including fever, cough, a nucleic acid test, body temperature recovery time) and physical function indicators (blood pressure, heart rate, body composition, muscle strength, joint mobility, activities of daily living) disappeared.Secondary outcome: The disappearance of the accompanying symptoms (such as myalgia, chest tightness, runny nose, headache, nausea, vomiting and diarrhea); COVID-19 nucleic acid test results for 2 consecutive times 
(not the same day) negative result rate, CT image improvement, common type to Incidence rate, clinical cure rate and mortality rate of severe illness.

Information sources: Search strategy - The search terms on PubMed are as follows: "Traditional Chinese exercise (eg, Taijiquan, Baduanjin, Wuqinxi, Yijinjing)" ;"COVID-19" OR "Corona Virus Disease 2019" OR "Novel Corona Virus" OR "2019- nCoV" ; "convalescence" OR "Rehabilitation" OR "recovery"; "randomized controlled trial" OR "randomized" OR "randomly" OR "clinicaltrial." A combination of medical subject headings (MeSH) and free text words will be used. The same search terms are used in other electronic databases. These search terms are shown in Table 1. Study selection - Documents will be retrieved according to the retrieval strategy. Before retrieval, all reviewers will discuss and determine the selection criteria, and then exclude some duplicate documents or documents with incomplete information. Finally, the document records will be imported into EndNoteX9 software for management. The above steps was performed independently by two researchers. Any disagreements will be resolved through discussions with the third researcher. Researchers will record all documents that do not meet the inclusion criteria and provide reasons for excluding them. We chose the PRISMA flow chart to show the entire process of selecting documents for the study.

Main outcome(s): Primary outcomes: The disappearance of the main symptoms (including fever, cough, a nucleic acid test, body temperature recovery time) and physical function indicators (blood pressure, heart rate, body composition, muscle strength, joint mobility, activities of daily living) disappeared. Secondary outcome: The disappearance of the accompanying symptoms (such as myalgia, chest tightness, runny nose, headache, nausea, vomiting and diarrhea); COVID-19 nucleic acid test results for 2 consecutive times (not the same day) negative result rate, CT image improvement, common type to Incidence rate, clinical cure rate and mortality rate of severe illness.

Quality assessment / Risk of bias analysis: The two researchers will use Cochrane's risk of bias tool to evaluate the methodological quality of the included studies. We will consider the following items: random sequence generation (selection bias); allocation hiding (selection bias); blinding of subjects and staff (performance bias); outcome evaluation blinding (detection bias); incomplete outcome data (Attrition bias); selective reporting (reporting bias); other sources of bias (other biases). The risk of bias in all aspects of the research is evaluated, divided into three levels: low risk, high risk, and unclear risk.

Strategy of data synthesis: Each result will be calculated and combined using RevMan 5.3. The specific implementation is based on the current version of the Cochrane Intervention System Evaluation Manual. If the heterogeneity test is not significant, the Mantel-Haenszel method is selected as the fixed effects model, and if statistical heterogeneity is observed ( $12 \geq 50 \%$ 或 $P<.1$ ), the random effects model is used. If the heterogeneity is substantial, we will make a narrative qualitative summary. We will clearly describe which studies are included and how to synthesize them as described. We will be transparent about the indicators used.

Subgroup analysis: We will conduct a subgroup analysis based on different patient characteristics, intervention methods (Traditional Chinese exercise), treatment duration and outcome indicators.

Sensitivity analysis: Sensitivity analysis is to analyze the quality of research, intervention methods, types, etc., and eliminate the researches with quality defects one by one, so that we can determine the source of heterogeneity.

Country(ies) involved: China. 
Keywords: COVID-19, Traditional Chinese exercise, Systematic review.

Contributions of each author:

Author 1 - Li Chen - Form analysis, Funding acquisition, Writing-original draft.

Email: lizi5225@qq.com

Author 2 - Yu Ji - Data curation, Form analysis, Software, Writing-review \& editing.

Email: mhwgo-jfy@qq.com

Author 3 - QiPeng Wang - Data curation, Form analysis, Methodology, Software, Writing-review \& editing.

Email: 937150039@qq.com

Author 4 - Peng Chen - Data curation, Software. 